\title{
Meine Studienreise nach Paris im Sommer des Jahres 1914
}

\author{
Hermann Schüttauf, mit einem Vorwort von Marcus Köhler
}

$\mathrm{D}$ er Name Hermann Schüttauf (1890-1967) ist mit vielen historischen Gartenanlagen im Osten Deutschlands verbunden. Der Gärtnerssohn aus Niederplanitz bei Zwickau gilt zurecht als ein Wegbereiter der deutschen Gartendenkmalpflege. Von 1929 bis zur Auflösung im Jahr 1949 war er Direktor der Staatlichen Gärten Sachsens und machte sich danach selbstständig. In dieser Funktion aber auch als Mitarbeiter in zahlreichen Gremien und Verbänden in der DDR wurde er immer wieder als Berater und Planer hinzugezogen, wenn es galt, historische Gärten zu erhalten, zu restaurieren und fortzuschreiben. Zudem wurde er im In- wie auch im Ausland mit Ausstellungen betraut, durch die er auch die zeitgenössische Entwicklung der Landespflege und des Gartenbaus darzustellen wusste. Seine Wurzeln konnte er dabei nicht verleugnen, da bereits seine Ausbildung den Kosmos gärtnerischer Möglichkeiten widerspiegelt: 1908/09 beim Hofgärtner Schulz in Schwerin angestellt, wechselte er in die Baumschule Poscharsky (Dresden) und absolvierte 1910 ein Praktikum beim bekannten Leipziger Gartenarchitekten Molsdorf, um danach - von 1911 bis 1913 - die Gartenbauschule in Dresden-Laubegast (später Pillnitz) zu besuchen. Mit dem Abschluss in der Tasche arbeitete er in Antwerpen im gerade neugegründeten Filialbüro des Mannheimer Gartenarchitekten Friedrich Brahe und bereiste danach England. Das Jahr 1914 kennzeichnet eine kurze Hospitantenzeit bei der Heimstätten AG Berlin-Nikolassee, in der Hermann Muthesius wirkte, und beim Gartenarchitekten Habich im Baubüro Korff in Laage/Mecklenburg. Der Blick in diese großbürgerlichen und internationalen Kreise mag bei Schüttauf den Wunsch hervorgerufen haben, weitere Erfahrungen im Ausland zu sammeln. Seine Bewerbung um ein Friedrich-August-Reisestipendium nach Frankreich wurde vom Vorstand der Gesellschaft für Botanik und Gartenbau (»Flora») positiv entschieden, so dass er zur besten Jahreszeit dorthin reisen und sich auch Werke der Gartenkunst anschauen konnte, die ihm später immer wieder als Referenzobjekte wichtig erscheinen sollten. In leitender Funktion konnte er später nicht nur selber Stipendien der »Flora« vergeben, sondern kümmerte sich auch um die Traditionspflege seiner ehemaligen Ausbildungsstätte in Laubegast/Pillnitz.

Da am 28. Juli 1914 Österreich-Ungarn Serbien den Krieg erklärte und innerhalb von wenigen Wochen der Erste Weltkrieg seinen Anfang nahm, sind Schüttaufs Aufzeichnungen nicht nur Schilderungen eines jungen reisenden Gärtners in jener Zeit, sondern ein Dokument der letzten Friedenstage eines zusammenbrechenden Europas.

Der Lebenslauf ist als Typoskript erhalten, das der Potsdamer Gartendirektor Harri Günther in seinen Forschungsunterlagen bewahrt hat. Freundlicherweise hat er sie dem Lehrgebiet zur Verfügung gestellt.

Marcus Köhler 
W ie mancher Besucher der Dresdener Gartenbauschule hat [er] den wohl alle 2 Jahre wiederkehrenden Besichtigungen des Schloßparkes zu Großsedlitz unter Führung seines in diesem Jahre dahingegangenen Lehrers und Meisters Bertram beigewohnt, hat seinen Erläuterungen gelauscht, ist mit ihm über breite Freitreppen, blumengeschmückte Parterres, durch schnurgerade, kulissenartig begrenzte Wege gewandert! Wie mancher hat ihm die Großzügigkeit der ganzen Anlage bewundert, sich über schöne, gut durchdachte Blicke und Perspektiven gefreut! Aber auch eine Sehnsucht hat er in fast allen seiner Schüler zu wecken gewußt! Und als der Elbdampfer mittags wieder heimwärts fuhr, da haben viele diese Sehnsucht weiter gesponnen: Einmal das große Urbild all dieser Schöpfungen in diesem Stil an Ort und Stelle zu besuchen, einmal aufzubrechen nach Versailles!

Und so habe ich mich aufgemacht nach Paris - just 3 Wochen vor Ausbruch des großen Völkerringens - ausgerüstet mit dem Reisestipendium der Königl. Sächs. Gesellschaft für Gartenbau \& Botanik »Flora« des Jahres 1914.-

Wenn mich nun Paris selbst anfangs enttäuscht hat, so mag das wohl daher gekommen sein, daß ich beim ersten Inaugenscheinnehmen der Stadt nichts fand, das mich besonders angezogen hätte! Ich fand in den Boulevards, in den Straßen und Plätzen der inneren Stadt wenig, das sich besonders hervorgehoben hätte von ähnlichen Stadtbildern in Berlin oder London!- Ein einziger Blick hat dieses Urteil dann aber umstoßen können: Der Blick vom Place de l'Étoile [heute: Place Charles-de-Gaulle] nach den Champs-Élysées! Eine derartig schöne und durchgebildete Achse besitzt weder Berlin noch London! Es sollte sich aber dann noch mehr finden, das mein anfängliches Urteil umstieß! Gelangt man die Champs-Elysées hinunter von der Place de l'Étoile bis zur prächtigen Avenue Alexandre III [Avenue Winston-Churchill], so eröffnen sich wiede- rum wundervolle Perspektiven, östlich auf die Place de la Concorde, westlich auf die Place de l'Étoile mit ihrem mächtig wirkenden Triumphbogen und schließlich südlich über die schöne Brücke Pont Alexandre III auf den Invalidendom mit seiner prächtigen, goldenen Kuppel. Geht man die Avenue des Champs-Élysées weiter hinunter zum schönsten Platz von Paris, dem Place de la Concorde, so muß man zunächst dessen gewaltige Dimensionen bewundern, seine architektonisch schöne Umrahmung anerkennen und dann: Auch hier wieder schöne Blicke, Perspektiven, die einen gefangen nehmen: Nördlich zwei in ihrer Gesamtheit harmonisch wirkende Prachtbauten, im Süden über die Pont de la Concorde bis zum Palais Bourbon und schließlich östlich und westlich wiederum die Achse vom Triumphbogen bis zum Louvre.- Ich denke ferner noch an den wunderbaren Blick vom Trocadéro mit seinen schönen, vorgelagerten Kaskaden über die leider etwas zu schmale Brücke (Pont d'Iéna) durch den Bogen des Eiffelturmes hinaus auf das Marsfeld mit seinen modernen Grünanlagen bis zur École Militaire.

Soweit Paris als Stadt selbst! Wenn ein Gärtner zu Studienzwecken nach der Seinestadt fährt, so will er in der Hauptsache wohl dasjenige dort studieren, was neben schönen Gebäuden, Straßen, Plätzen für ihn besonders bemerkenswert ist: Er will sich mit der Gartenkunst eines Lenôtre, seiner Vorgänger und Nachfolger vertraut machen! Und so darf ich denn den Park von Monceau mit seinem Reichtum an schönen Denkmälern und den an und für sich sehenswerten Park Buttes-Chaumont, der dem Techniker mehr Interesse ablocken dürfte als dem Künstler, übergehen und mich zunächst dem Luxemburg-Garten zuwenden.

Ein Garten, der wohl als ein Meisterstück französischer Gartenkunst einzuschätzen ist. Den größten Eindruck machte auf mich die großzügig und großartig durchgeführte Mittelachse, eigenartig auch deswegen, weil sie über den eigentlichen Park selbst in langen Baumreihen mit einem Schmuckstrei- 
fen in der Mitte weit hinaus geführt worden ist.- Ein tiefer gelegener Blumengarten mit einem riesigen, achteckigen Wasserbecken lagert sich der Gartenseite des schönen Luxemburg-Palais vor. Trotz seiner Abgeschlossenheit fügt sich dieser Blumengarten dem ganzen Park gut ein! Auffallend ist die Größe der Kiesflächen; man ist versucht die Frage aufzuwerfen, ob der Garten als Privatbesitz oder öffentliche Grünanlage gedacht ist!- Dies' Empfinden habe ich in allen Schöpfungen des französischen Gartenstiles, besonders in Versailles, dann aber auch in Saint-Cloud und zum Teil auch in Chantilly gehabt. Obwohl alle diese doch nur als Privatgärten angelegt worden sind, so eignen sie sich doch heute vorzüglich als Volksgärten in weitgehendem Maße!

Im Luxemburg-Garten gefiel mir noch besonders die »Fontaine Médicis«.- In dem unregelmäßig angelegten Teil des Gartens fand [sich] auch die eigentümliche Randbepflanzung der Gehölzgruppen mit Sommerblumen wieder, die man in den Gärten und Parks von London so reichlich sieht. Was mir aber dort gefiel hätte ich hier lieber vermißt. Was dort in üppiger Pracht, in breiten sonnigen Blumen- und Farbenklexen zu sehen war, erschien mir hier kümmerlich, kleinlich und schablonenhaft!

Einen ebenfalls schönen und eigenartigen Blick im Luxemburg-Garten möchte ich nicht unerwähnt lassen: der Blick vom $\mathrm{Pa}$ lais nach dem Pantheon, das man zwischen Baumkulissen und Grünstreifen im Endpunkt der Achse liegen sieht, eigenartig deshalb, weil sich der Architekt das Bauen einer Achse nicht entgehen ließ, die sonst eigentlich gar nicht in die übrige Aufteilung des Parkteiles paßt!

Ein weiteres Ziel war das Schlößchen Bagatelle mit seinen Parkanlagen. Bei meinem langen, [zunächst] vergeblichen Suchen in diesem Parke nach dem Schlößchen selbst kam ich durch einen ziemlich ausgedehnten landschaftlichen Garten mit gut gepflegten Wiesen, Weihern mit prächtigen Wasserpflanzen, Grottenbauten und schö- nen Bäumen. Ich fand bei dieser Wanderung aber auch Bilder, die weniger schön, dafür umso eigenartiger zu nennen sind, so z.B. künstliche Ruinen oder Obelisken.- Ja sogar durch einen künstlichen Tunnel, den man auf ebener Erde gesetzt und mit einem Erdwall, der dicht bepflanzt war, überdeckt hatte, führte mich mein Weg.- Auch zwei Rosengärten entdeckte ich: einen kleineren in langgestreckter Form, von hohen Mauern umschlossen, die mit Kletterrosen und Clematis regellos berankt waren. Durch die Mitte des Rosengartens führte ein geradliniger Weg, der abwechselnd von Rosen und Glyzinenbögen überspannt war. $\mathrm{Zu}$ beiden Seiten dieses Weges blühten auf kleinen Beeten in buntem Durcheinander Stauden aller Art!

Der größere dieser beiden Gärten war nur mit Rosen bepflanzt, die in allen Formen gezeigt wurden: niedrige Gruppenrosen, Hochstämme, Trauerrosen an Säulen, Laubengängen oder einfachen, aber gut wirkenden Bögen.- Wenig gefallen hat mir das der Orangerie vorgelagerte kleine Parterre infolge seiner zerrissenen Form und unruhig wirkenden Aufteilung.

Das Schlößchen selbst nun war des Suchens wert. Ein kleines aber reizendes Gebäude, vor dessen Gartenfront ein langer, regelmäßig angelegter Gartenteil sich aufbaut: ein etwas vertieftes, langgestrecktes Rasenparterre mit Blumenrabatten an den Längsseiten. Das Ganze war von außerordentlich wohltuender und ruhiger Wirkung! Das bewiesen auch die vielen, gut besetzten Bänke längs des Parterres!

Einen ebenfalls schönen Blick möchte ich nicht unerwähnt lassen: Der Eingang zum Schloß - oder besser zum Schloßhof. Unter dem Schatten zweier mächtig hoher Platanen grüßen uns zwei kleine Pförtnerhäuschen, an die sich rechts und links mit Efeu völlig verkleidete Mauern in kreisrunder Form anschließen.

An den Besuch des Schlößchens Bagatelle hab ich dann eine Wanderung durch den Bois de Boulogne angeschlossen. Etwas besonders Bemerkenswertes habe ich aber in 
dem Walde nicht entdecken können! Seine riesige Ausdehnung (878 ha), seine gut gepflegten Straßen und Wege, sein schöner waldartiger Eichenbestand, das wäre eigentlich alles, was mir aufgefallen ist. Ich bin sogar der Ansicht, daß der an und für sich wenig gepflegte Hyde-Park dem Londoner mehr biete mit seinen ausgedehnten Wiesenflächen und seinen lichten Hainen als das Bois de Boulogne mit seinen dichten, schönen Waldungen und gut gepflegten Wegen dem Pariser - wenigstens in Bezug auf die wenig oder gar nicht bemittelten Großstadtkinder.

\section{Chantilly}

Als erster Park, dessen Schöpfer Lenôtre selbst ist, hatte ich Chantilly in meinem Reiseprogramm vorgesehen!

Wenn man über die breite Rennbahn von Chantilly kommt, sieht man das Schloß schon von weitem liegen mit seinen Türmen und Türmchen. Ein Schloß, das in seiner Unregelmäßigkeit, seinen vorspringenden und zurückfallenden Bauten, umgeben von breiten Wassergräben einen entzückenden, "landschaftlichen " Blick abgibt. Dieses Unregelmäßige, »Landschaftliche« läßt sofort die Frage auftauchen, wie ein Lenôtre mit seinem streng symmetrischen Aufbau des Parkes hier Meister geworden sein mag.Und steht man dann auf der breiten Schloßterrasse und läßt den Blick über das große Rasenparterre zu Füßen, über den in der Achse etwas ausgebuchteten Kanal bis hinauf in die sich nach und nach enger schließenden Laubmassen schweifen, so muß man den Künstler, der das geschaffen, bewundern! Die Achse selbst ist prächtig durchgeführt! Nur schien es mir, als ob der organische Zusammenhang zwischen Schloß und Park, die rhythmische Einheit dieser beiden Faktoren fehlte - wenigstens aber lange nicht die Vollendung erreicht, wie im Luxemburg-Garten oder gar in Versailles.- Vortrefflich hat man es aber auch hier verstanden, die weite Landschaft in den Park einzubeziehen. Der eigentliche Schloßgarten hört mit dem das Rasenparterre ab-

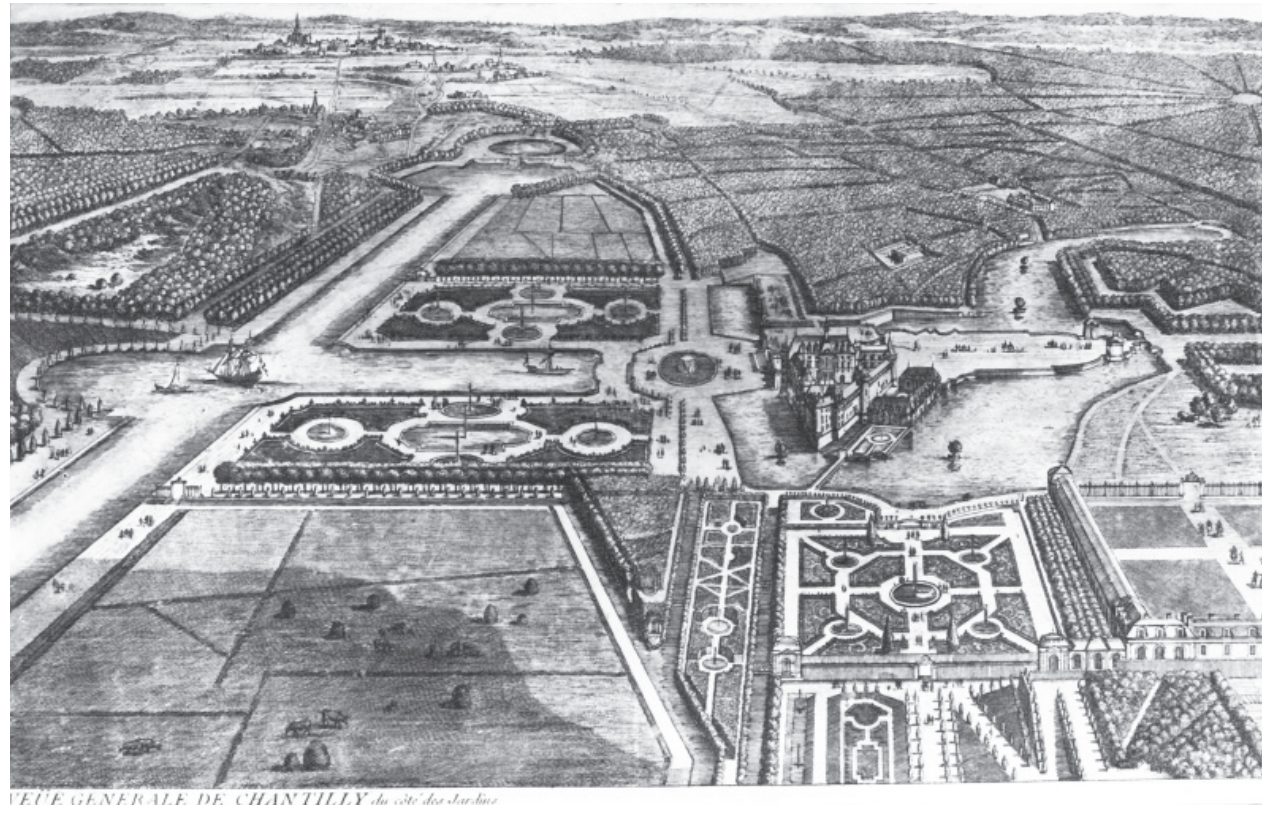

1 | Schloss und Schlosspark Chantilly, Gesamtübersicht, Radierung, unbekannter Künstler um 1670/vor 1703 (SLUB, Deutsche Fotothek, Heinz Nagel, Staatliche Kunstsammlungen Dresden (SKD), Kupferstich-Kabinett). 
schließenden Kanal auf, und welch' großartige Tiefenwirkung ist hier erzielt worden! Man spürt die gestaltende Hand des Künstlers auch jenseits des Kanales und sieht, wie geschickt er seine Achse fortzuführen weiß. In diesem Blicke von der Terrasse aus, sind Garten und Landschaft ein großes Ganzes!Durch ihre Schönheit bemerkenswert sind auch die beiden das Rasenparterre seitlich abschließenden hohen Platanenalleen. Weiter fand ich inmitten des "landschaftlich " bearbeiteten Teiles eine in einem Wassergraben eingebaute Insel, l'Ile d'Amour, die in ihrem streng geometrischen Aufbau mit einem kunstvoll aus Holzlattenwerk gearbeiteten Pavillon (als Abschluß der Längsachse) und breiten, von Steinvasen und Wasserbecken unterbrochenen Taxushecken (als Seitenbegrenzung), ein Teilchen für sich bildete.

Lange Zeit bin ich in dem sich westwärts anschließenden, prächtigen, waldartigen Parkteile herumgewandert, ohne auf große Kunstwerke zu stoßen. Und doch waren es Stunden des Genießens: Am Himmel die an diesem Tage brennend heiße Sonne und drinnen an den einsamen, schnurgeraden, heckenartig von hohen Laubwaldbestand eingerahmten Wegen Schatten und angenehme Kühle! Ab und zu stößt man auf freie, ausgesparte Rasenplätze, die durch die unten heckenartig aufgeputzten, oben aber ihrem natürlichen Wachstum überlassenen Bäume diskret nach außen abgeschlossen sind und mir so recht zum Erholen oder Spielen jeglicher Art geeignet erschienen.

Was in Chantilly aber den stärksten Eindruck auf mich gemacht, ist der Etang de Comelle (wenn ich mich nicht im Namen irre), der westliche Abschluß des Canal de la Marche: ${ }^{1}$ Ein riesiger regelmäßig viereckiger Teich, umgeben ringsum von einem breiten Rasenteppich, durch den ein Fahrweg läuft, und dann schließt sich eine etwa 20 m hohe, heckenartige Laubwand an. Das war alles! Ohne jegliche architektonische Zierde, ohne jeden figürlichen Schmuck und dennoch: welche großartige Wirkung! Die breite Wasserfläche und die sich auf derselben spie- gelnden Heckenwände waren die einzigen Mittel, die hier verwendet worden waren. Ich werde nie an Chantilly denken mit seinen mannigfachen Schönheiten, ohne mich dieses herrlichen Teiles zu erinnern!

\section{Saint-Cloud}

Beim Besuche von Saint-Cloud habe ich bedauert, daß ich keinen Plan vom Parke zur Hand hatte! Ich fand die Gliederung und Aufteilung des Geländes bei weitem nicht so straff und scharf wie in den übrigen besuchten Gärten und Parks! Ebenfalls war hier die Orientierungsmöglichkeit keine allzu große, ein Vorzug, der sonst doch den meisten französischen Gärten eigen ist. Das mag zum Teil an der natürlichen Beschaffenheit des Geländes gelegen haben - ein großer Teil des Parkes liegt auf einem steilen Abhang - und außerdem fehlt jetzt einer der Hauptfaktoren - der z.B. in Versailles souverän herrscht - das Schloß selbst. Befindet man sich auf dem Platze, wo bis 1870 das Schloß gestanden hat, so fallen wiederum 2 Achsen sofort in die Augen: die eine weniger durchgebildete südlich, die ihre Fortsetzung in der Allée du Fer à Cheval hat, die andere westlich. Diese letztere ist die Hauptachse, die mehrfach abgestuft hinauf die Höhen führt. Der betonteste Teil dieser Achse erstreckt sich vom Schloßplatz über eine breite Freitreppe zum Parterre de l'Orangerie mit blumengeschmückten Rasenbahnen und Springbrunnen, von da über 3 weniger breite Treppen zu einem langgestreckten, sanft aufsteigenden Blumenparterre, das mit Marmorfiguren reich geschmückt ist, zum "Bassin des 24 Jets«, einem großen, runden Platz mit ausgedehnten Wasserflächen. Von hier führt uns wieder eine breit angelegte Rasenbahn in etwas erhöhterer Steigung zu einem großen, kreisrunden Wasserbassin. Die Achse selbst setzt sich in einer langen Allee fort. Als scharfe und doch weich erscheinende Seitenbegrenzung dieser eben geschilderten Achsenteile denke man sich nun wieder dichten Laubwald, dessen Grenzbaumreihe unten heckenartig aufge- 
putzt ist und oben in Wildwuchs übergeht.

Architektonisch reizvoll ist auch der Blick vom südlichen Eingang über das "Bassin du Fer à Cheval« hinweg zum Schloßplatz. Wieviel schöner muß aber gerade dieser Blick gewesen sein zu Zeiten, als das Schloß selbst noch der Kernpunkt in diesem Blick war! Der Besucher von Saint-Cloud findet in den übrigen Teilen des Parkes breite schneisenartige Wege, deren guten Zustand man allerdings nicht rühmen kann. Erwähnenswert sind noch die "Hautes Cascades « [Grande Cascade], die in ihrer sonst guten Tiefenwirkung mir ziemlich um die Hälfte zu schmal erschienen!

Abschied von Saint-Cloud habe ich genommen von der Anhöhe aus, die unmittelbar am Schloßplatz steil hinaufsteigt, habe hinunter gegrüßt ins Seinetal, hinüber nach der Weltstadt, mit dem Bois du Boulogne und hinauf nach dem drohend ins Land lugenden Mont Valérien!- Kehrt man von die- ser Stelle aus nach der Stadt Saint-Cloud zurück, so kommt man durch ein Stück vorzüglich gepflegten Garten - zum großen Unterschiede von den übrigen Teilen des Parkes - mit kurz gehaltenem saftgrünem Rasenteppich, Blumenbeeten in einer Pracht, wie ich sie sonst auf der ganzen Reise nicht zu sehen bekam - kurzum ein Schmuckkästlein in mancher Hinsicht! Aber gib' acht, Wanderer! Hier darfst du nicht auf den Rasen treten, auch kein Papier wegwerfen - angebrachte Tafeln verkünden dies mit großen Lettern - und mit Recht: Man befindet sich hier in einem Teile des Parkes von Saint-Cloud, der im englischen Landschaftsstile angelegt war!-

\section{Versailles}

Das Schloß und die Gärten von Versailles hatte ich mir bis zuletzt aufgehoben, und mit den hochgespanntesten Erwartungen bin

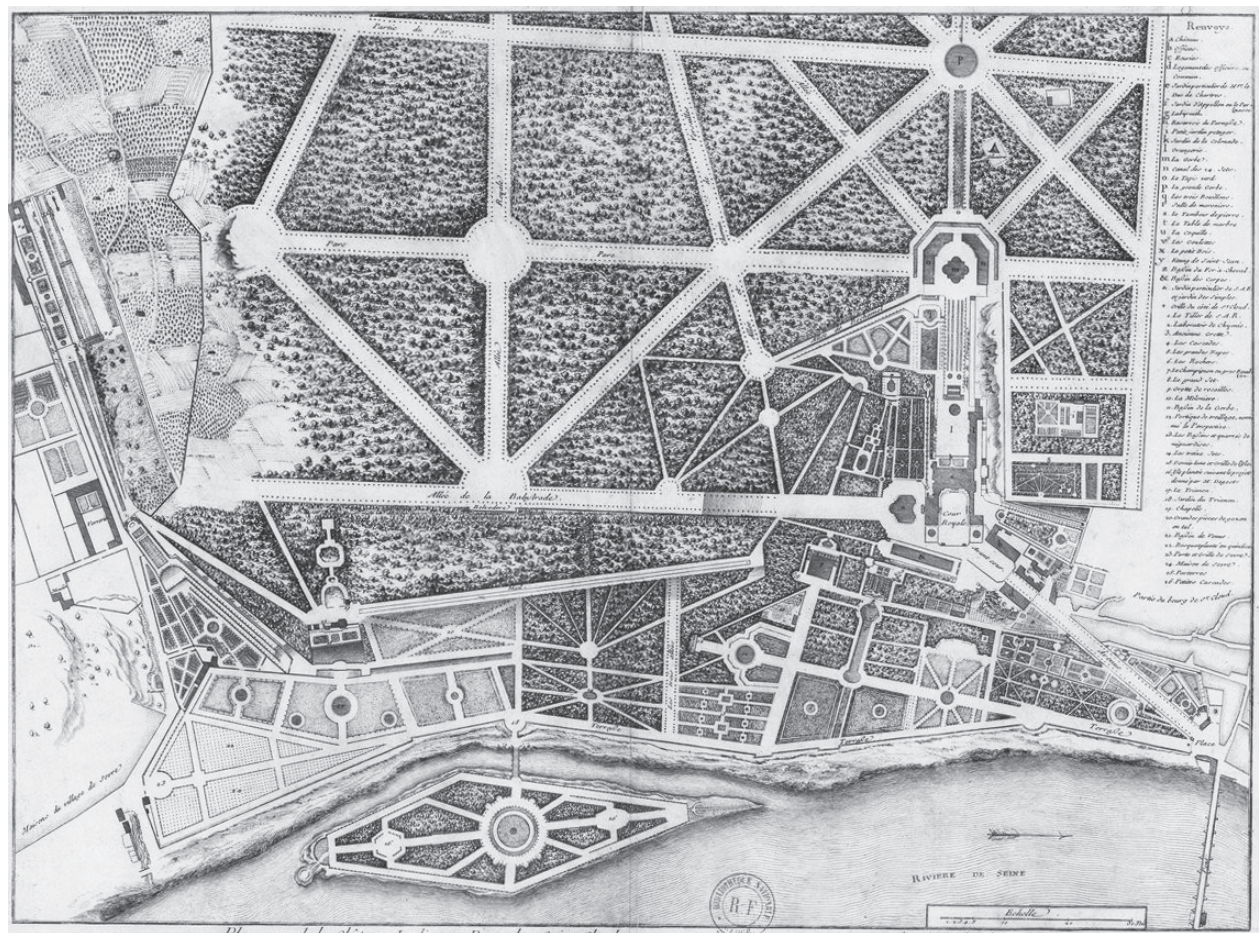

2 | Übersichtsplan von Schloss und Schlosspark Saint-Cloud, Radierung, J. Mariette 17. Jh. (Bibliothèque nationale de France, département Cartes et plans, GE C-1395). 
ich denn eines Morgens hinausgefahren nach dieser Stätte Lenôtre'schen Schaffens!

Durch eingehendes, vorheriges Studium der Planung war ich ziemlich genau über die Anlage unterrichtet. Und wenn ich mir danach wohl ein zutreffendes Bild im Großen und Ganzen zu machen geglaubt hatte, so bewies ein Blick auf die westliche Hauptachse das Gegenteil!- Ich habe lange Zeit auf der obersten Schloßterrasse gestanden, um dies Bild ja recht gut aufnehmen zu können. Überraschend war für mich zunächst die großartige Terrassierung von Wasserparterre zum großen Blumenparterre mit dem „Bassin de Latone«, ferner die prächtige Tiefenwirkung die Achse hinunter über den langen Kanal hinweg bis - ja, hier gibt es eben keine Grenzen. Nur eingeengt wird der Blick ein wenig durch zwei rechts und links stehende Pappeln, die in ihren Grenzlinien so angenehm weich erschienen. Und wenn sonst Lenôtre - dem Gärtner wohl eigentlich gar nicht zu gefallen - Baum und Strauch in seiner Eigenart dem Besucher seiner Gärten wenig oder gar nicht zeigt, ja meistens gewaltsam unterdrückt - in Versunkensein dieses Blickes mit der schönen Wirkung dieser beiden Pappeln kann man sich mit ihm aussöhnen - ich habe es getan!

Die beiden andern Achsen führen von der Schloßterrasse aus nach Süden und Norden. Die südliche zeigt uns zunächst das Südparterre (mit eigenartigen, streng geometrischen Grundrißformen), dann das Orangerieparterre und mündet im Schweizerbecken, das von einem dichten Kastanienwald wirkungsvoll umrahmt wird. Die nördliche Achse führt zum Nordparterre, durch die "Wasserallee« zum Drachenbecken und schließt mit dem großen Neptunbecken ab. Beim genaueren Studium dieser 3 Achsen lernt man wohl erkennen, daß hier ein Künstler tätig gewesen ist! Man bewundert, wie sich hier Lenôtre über ansehnliche Terrainunterschiede hinweggesetzt hat; wie er sie umgekehrt in hervorragender Weise benutzt hat beim Aufbau seiner breiten Terrassen, die sich wiederum dem Ganzen in ihrem Maßen als ein wohl abgestimmter Teil einfügen. Man merkt auf Schritt und Tritt, daß hier ein starker Wille seinen künstlerischen Ideen alles unterwarf, um das zu schaffen, was die Nachwelt bis zu uns bewundert und anerkannt hat. Ausgesprochen darf aber wohl werden, daß kein Künstler jemals wieder so aus dem Vollen hat schaffen können wie Lenôtre, daß keinem wieder solch' unerschöpfliche Mittel an Kapital und Arbeitskräften und vielleicht auch an künstlerischen Mitarbeitern zur Verfügung [gestanden] haben wie dem großen Baumeister Ludwigs des 14. bei der Schaffung von Versailles!

Genußreiche Stunden habe ich in dem schattigen, waldartigen Teilen des Schloßparkes zugebracht. Ich habe gestaunt, auf wie mannigfaltige Art die einzelnen Kreuzungspunkte der Alleen und Wege, die auf dem Plane schließlich sich sehr einander gleichen, behandelt sind, wie anheimelnd doch die einzelnen »Gartenräume« wirken und wie gewählt einzelne dekorative Plastiken, Hermen, Vasen verwendet sind! Größere architektonische Kunstwerke, auf die man unerwartet stößt, wie der Marmorhof der »Kolonnaden « oder das "Bosquet des Dômes « oder die so verschieden behandelten, großen Wasseranlagen wie das »Bassin de Latone", das "Bassin d'Apollon «, das "Bassin de Neptune« wecken das Interesse des Wanderers stets von neuem, wenn sein Geist sich in beschaulicher Ruhe dem Genusse der immer und immer wiederkehrenden, schönen, dichten, schattigen Baumgänge hingeben will! Auch für das streng Geradlinige, Architektonische im Weg, Platz, Pflanzung findet man Abwechslung: Wie reizvoll wirken in ihrer Unregelmäßigkeit das Bosquet des Königs und die Bäder des Apollo!-

Am nördlichen Arme des großen Kanals grüßt den Besucher Groß-Trianon, das dem Gärtner manches biete, an dem er sich freuen und auch lernen kann! - Ein überraschend farbenprächtiges Bild eröffnet sich, wenn man durch die Säulen im Mittelbau des Schlößchens tritt. Auf einem großen, quadratischen Hofe mit zwei Wasserbecken 
wetteifern die Blumen in ihren lachenden Farben mit dem prächtig rotbunten Marmor des Schlosses, eines feinen Renaissancebaues, flachgeschossig mit hohen, schönen Fenstern und Türen. Hier wird der Besucher so freudig gestimmt durch die leuchtenden Farben, daß er sich schwer losreißen kann von dem heiteren Bilde, das noch besonders vornehm durch die grünen, dichten Baumkronen der Umgebung wirkt! Der sich anschließende Park mit seinem dichten Laubdache, streng regelmäßig angelegt, erinnert in seinen schattigen Alleen, seinen Brunnen und Wasserbecken an Versailles. An schlechter, mangelhafter Pflege übertrifft er seinen großen Bruder aber bei Weitem!

Wer weiter wandert, schaut dann noch einmal all' das Schöne französischer Gartenkunst, all' das typische der Schule Lenôtres: Klein-Trianon! In Versailles wirkt das Gigantische, Machtverkörpernde, Großzügige im Schloß, in den Gärten auf uns ein. Hier findet man mehr das »Idyll«! Jeder Winkel und jedes Teilchen zeigt eine liebevolle Durcharbeitung: das kleine Schlößchen, die blumengeschmückten Terrassenwinkel, der sich anschließende französische Garten mit seinen guten Abmessungen und seinen eine schöne Raumwirkung schaffenden, geschnittenen Bäumen! Hier darf man die Behauptung wohl aussprechen, daß wir Schöneres auf dem Gebiete der französischen Gartenkunst kaum erwarten dürfen!

1 Es ist nicht eindeutig, auf welchen Ort sich Schüttauf hier bezieht: Die erwähnten Étangs de Commelles dürfte er eher nicht gemeint haben. Diese Abfolge von Teichen befindet sich ca. $4 \mathrm{~km}$ südlich des Schlosses im Wald von Chantilly und passt nicht zur nachfolgenden Beschreibung. Wahrscheinlich hatte er die Partie nördlich des Pavillon de Manse im Sinn. Hier, am westlichen Abschluss des Grand Canal und des Canal Saint Jean befand sich ein großes, viereckiges Wasserbecken, das als Reservoir für die Wasserspiele des Schlossparks diente und über
Der weitere englische Park von KleinTrianon, der - nach meinem Führer sich wohltuend von den beschnittenen Hecken des Versailler Gartens - sagen wir: von den gartenkünstlerischen Denkmälern in Versailles -, abheben sollte, sei nur seines Daseins wegen erwähnt! Auf mich konnte er nach all' dem Gesehenen und Durchlebten keinen Eindruck mehr machen!-

Manchem habe ich in Versailles, SaintCloud, Chantilly und den anderen allen skeptisch gegenüber gestanden, manches hat hier meinem Gefühl bei aller Bewunderung und Anerkennung - widersprochen und manches hat mich schließlich nicht recht erwärmen können! Für eine Sache muß aber jedem das Herz aufgehen, der Schöpfungen Lenôtres, seiner Vorgänger und Nachfolger gesehen hat: wie hervorragend man es verstanden hat, diesen Gärten in ihren kleinsten Bauteilchen, sei es Baum und Strauch - sei es toter Stein, dies aufzuprägen: Hier haben Menschen die Natur in jeder Art und Weise zu meistern gesucht, haben die Schönheiten der Natur nicht nachahmen, sondern selbst schöpferisch tätig sein wollen.

Beides haben sie erreicht: Heute nach 200 Jahren noch sieht der Laie, daß der Mensch mit seinem Willen hier geschaffen haben muß - der Fachmann, daß er wahre, echte Kunst bewundern darf!

(Textedition: Jonathan Sironi, Janis Vetter) den Canal de la Machine und den Canal de Manse angebunden war (Bibliothèque du musée Condé, château de Chantilly, Album du Comte du Nord, planche 25 "Les grandes cascades, Fontaine minérale et Pavillon de la Machine hydraulique«, 1784, Mairie de Chantilly (Hg.): Parcours Chantilly. Le long des canaux, in: http://www.oisetourisme.com/ randopic060v504rlu/chantilly-le-long-des-canaux/ chantilly (Stand: 29.01.2018); https://fr.wikipedia. org/wiki/Pavillon_de_Manse (Stand: 29.01.2018)). 